\title{
Homogenization via $p$-FEM for Problems with Microstructure
}

\section{Report}

Author(s):

Matache, Ana-Maria; Schwab, Christoph

Publication date:

1999-04

Permanent link:

https://doi.org/10.3929/ethz-a-004288545

Rights / license:

In Copyright - Non-Commercial Use Permitted

Originally published in:

SAM Research Report 1999-09 


\section{Homogenization via $p$-FEM for Problems with Microstructure}

A.M. Matache and C. Schwab

Research Report No. 99-09

April 1999

Seminar für Angewandte Mathematik

Eidgenössische Technische Hochschule

CH-8092 Zürich

Switzerland 


\title{
Homogenization via $p$-FEM for Problems with Microstructure
}

\author{
A.M. Matache and C. Schwab
}

Seminar für Angewandte Mathematik

Eidgenössische Technische Hochschule

CH-8092 Zürich

Switzerland

Research Report No. 99-09 April 1999

\begin{abstract}
A new class of $p$ version FEM for elliptic problems with microstructure is developed. Based on arguments from the theory of $n$-widths, the existence of subspaces with favourable approximation properties for solution sets of PDEs is deduced. The construction of such subspaces is addressed for problems with (patch-wise) periodic microstructure. Families of adapted spectral shape functions are exhibited which give exponential convergence for smooth data, independently of the coefficient regularity. Some theoretical results on the spectral approach in homogenization are presented. Numerical results show robust exponential convergence in all cases.
\end{abstract}




\section{Microstructure and $n$-widths}

Numerous problems in engineering and the sciences involve media with small-scale features, such as arrays of rivets, stiffeners, fibers or porous media etc. Typically, the solutions of such problems vary on multiple scales, e.g. on a large scale which is the size of the domain $\Omega$ and on a small scale $\varepsilon$ such as the distance of the spatial inhomogeneities, see Fig. 1. In many cases scale resolution, i.e. the resolution of the smallest solution scale with numerical methods, is not feasible due to computational expense. This is particularly so when higher order elements are used.

If the microstructure is periodic the theory of homogenization gives a macroscopic description of the limit when the small length scale $\varepsilon$ tends to zero. The resulting homogenized solution can be easily computed but does not exhibit the microscale anymore, (5), (6). This microscale can be recovered by so-called correctors in homogenization which are in many cases not useful for numerical purposes. To compute higher order terms in asymptotic homogenization expansions with respect to $\varepsilon$ is not an alternative either because inclusion of these terms need not improve the accuracy at fixed, positive $\varepsilon$ and these terms may not even exist for rough data.

We propose therefore here a different approach designed to overcome the above mentioned pitfalls. The main idea is to replace the polynomials by function spaces that are, in a sense, adapted to the coefficients of the elliptic operator. The basic understanding for our approach can be derived from the theory of $n$-widths, initiated by Kolmogoroff.

For a linear space $X$ with norm $\|\circ\|_{X}$ and for a subset $S \subset X$ the Kolmogoroff $n$-width is given by

$$
d_{n}(S, X)=\inf _{V_{n}} \sup _{f \in S} \inf _{g \in V_{n}}\|f-g\|_{X}
$$

the first infimum being taken over all subspaces $V_{n} \subset X$ of dimension $n$.

Let $\Omega \subset \mathbb{R}^{d}$ be a bounded, open set and consider the Dirichlet problem

$$
L u:=-\nabla \cdot(A(x) \nabla u)+a_{0}(x) u=f \text { on } \Omega,\left.\quad u\right|_{\partial \Omega}=0,
$$

where the conductivity matrix $A(x) \in L^{\infty}(\Omega)^{d \times d}$ is symmetric positive definite and $a_{0}(x) \in L^{\infty}(\Omega)$ is uniformly positive at a.e. $x \in \Omega$. The variational formulation of (1.2) is: find $u \in H_{0}^{1}(\Omega)$ such that

$$
B(u, v)=F(v) \quad \forall v \in H_{0}^{1}(\Omega)
$$

and its Finite Element discretization reads: find $u_{n} \in V_{n}$ such that

$$
B\left(u_{n}, v\right)=F(v) \quad \forall v \in V_{n} .
$$

Here $V_{n} \subset H_{0}^{1}(\Omega)$ is an $n$-dimensional subspace. The FE-solution $u_{n}$ is optimal in the energy norm of the problem:

$$
\left\|u-u_{n}\right\|_{E} \leq \inf _{v \in V_{n}}\|u-v\|_{E}
$$

Therefore, efficient FEM are based on the proper design of $V_{n}$ with respect to the anticipated solution class $S$ of the problem. The Kolmogoroff $n$-width $d_{n}$ gives us the best possible rate of convergence in the energy norm $\|\circ\|_{X}=\|\circ\|_{E}$ achievable with any subspace sequence $\left\{V_{n}\right\}_{n}$ with $\operatorname{dim} V_{n}=n$. There holds the following theorem.

Theorem 1.1 Let $\Omega \subset \mathbb{R}^{d}$ be a bounded domain with Lipschitz boundary $\partial \Omega$ and assume that $\Omega \subset G \subset$ $\mathbb{C}^{d}$ with strict inclusions for some open neighborhood $G$ of $\Omega$ in $\mathbb{C}^{d}$. Assume that $f \in \mathcal{U}^{G}$, where

$$
\mathcal{U}^{G}:=\left\{f \in L^{\infty}(G) \mid\|f\|_{L^{\infty}(G)} \leq 1, f \text { holom. in } \bar{G}\right\} .
$$




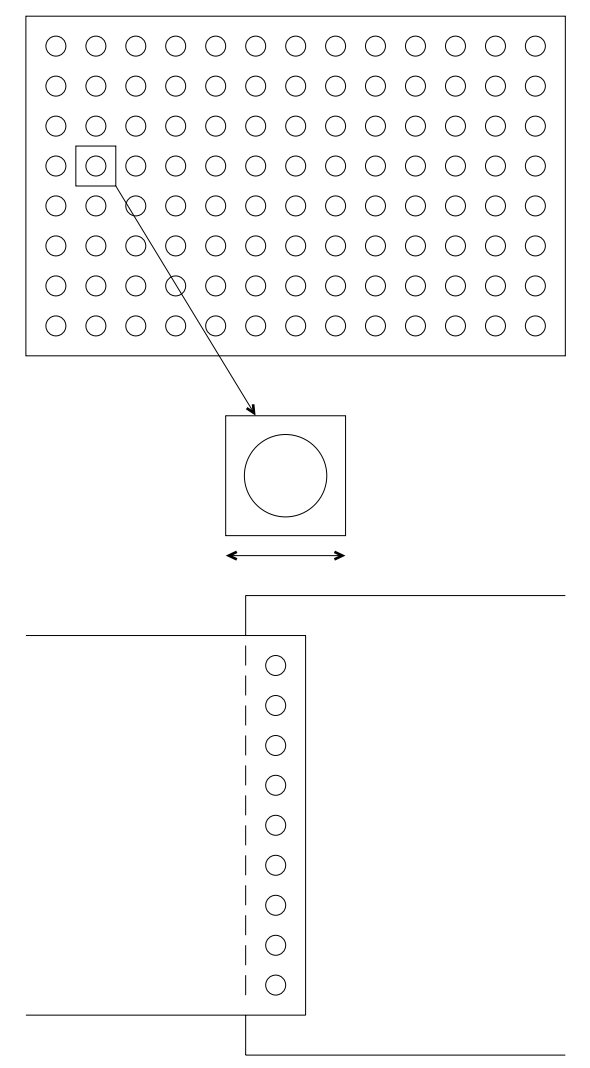

Figure 1: Domains with periodic, resp. locally periodic structure.

Assume that $A(x) \in L^{\infty}(\Omega)^{d \times d}$ and $a_{0}(x) \in L^{\infty}(\Omega)$ in (1.2) are positive. Then the solution operator $T: H^{-1}(\Omega) \rightarrow H_{0}^{1}(\Omega)$ to $(1.2)$ is bijective and we define the set $S$ in (1.1) to be

$$
S=T\left(\mathcal{U}^{G}\right) .
$$

Then there holds

$$
C_{1} \exp \left(-r_{1} n^{1 / d}\right) \leq d_{n}\left(T\left(\mathcal{U}^{G}\right), E\right) \leq C_{2} \exp \left(-r_{2} n^{1 / d}\right),
$$

where $C_{i}$ and $r_{i}>0$ depend only on $\Omega$ and $G$ and on the ellipticity constants of the bilinear form $B(\cdot, \cdot)$.

For a proof of this theorem, we refer to (9). From the proof it is clear that results of the type of Theorem 1.1 hold for practically all well-posed elliptic boundary value problems. We see in particular that exponential convergence is achievable even if the coefficients $A(x), a_{0}(x)$ and the boundary $\partial \Omega$ are very irregular. The global regularity of the solution in standard Sobolev spaces is very low in these cases and standard FEM based on piecewise polynomials will only give poor convergence rates.

The impact of Theorem 1.1 on numerical computation depends, of course, on whether sequences $\left\{V_{n}\right\}_{n}$ of subspaces, for which the bounds (1.7) are attained, can be obtained cheaply. Such subspaces are necessarily problem adapted. Some choices for elliptic problems in two dimensions with stratified quasi one-dimensional coefficients have been proposed in (2). Here, we present a methodology for determining such subspaces for elliptic problems in divergence form with periodic, oscillating coefficients. Such 
homogenization problems have been well investigated by asymptotic analysis (see, e.g. $(5 ; 6)$ and the references there). It turns out, however, that the information on the solution obtained by asymptotic analysis is insufficient to construct $\left\{V_{n}\right\}_{n}$ satisfying (1.7) (7). For the construction of such $\left\{V_{n}\right\}_{n}$, we therefore present first a more general, non-asymptotic approach to homogenization due to (3).

\section{Homogenization}

\subsection{The homogenization problem}

We consider now a particular case of (1.2), namely that the coefficients $A(x)$ and $a_{0}(x)$ have the special form $A(x / \varepsilon), a_{0}(x / \varepsilon)$, where $A(y), a_{0}(y)$ are $2 \pi$ - periodic in each variable, i.e.

$$
A(y)=A\left(y+2 \pi \mathbb{Z}^{d}\right), \quad a_{0}(y)=a_{0}\left(y+2 \pi \mathbb{Z}^{d}\right),
$$

and $\varepsilon>0$ is small, i.e. we are in the setting of (classical) homogenization. If $A$ is piecewise constant, (1.2) models, for example, the matrix and the fibers of a composite or a bi-material mixture. The right hand side $f$ is assumed to be analytic in $\bar{\Omega}$ (this is essential for exponential convergence, but spectral convergence results can be proved if the regularity of $f$ is finite $(7),(8))$. We denote the solution of (1.2) by $u^{\varepsilon}(x)$ in order to underline the dependence on $\varepsilon$.

We consider (1.2) on the unbounded domain $\Omega=\mathbb{R}^{d}$ and assume that

$$
\eta \top A(x) \eta \geq \gamma|\eta|^{2}, \quad a_{0}(x) \geq \gamma>0, \quad \forall \eta \in \mathbb{R}^{d} \quad \text { at a.e. } x \in \mathbb{R}^{d},
$$

with the condition $\lim _{|x| \rightarrow \infty} u^{\varepsilon}(x)=0$.

Next, for $\nu \in \mathbb{R}$ and $j=0,1$ we introduce the weighted Sobolev spaces $H_{\nu}^{j}\left(\mathbb{R}^{d}\right)$, defined as the completion of $C_{0}^{\infty}\left(\mathbb{R}^{d}\right)$ with respect to the $\|\cdot\|_{j, \nu}$ norm: $H_{\nu}^{j}\left(\mathbb{R}^{d}\right):=\overline{C_{0}^{\infty}\left(\mathbb{R}^{d}\right)}\left\|^{\|}\right\|_{j, \nu}$, where for $u \in C_{0}^{\infty}\left(\mathbb{R}^{d}\right)$ we define

$$
\|u\|_{j, \nu}^{2}=\int_{\mathbb{R}^{d}} \sum_{|k| \leq j}\left|D^{k} u(x)\right|^{2} e^{2 \nu|x|} d x .
$$

For a variational formulation of $(1.2)$ on $\Omega=\mathbb{R}^{d}$, for each $\varepsilon>0$ we consider the sesquilinear form $\Psi(\varepsilon)[\cdot, \cdot]: H_{-\nu}^{1}\left(\mathbb{R}^{d}\right) \times H_{\nu}^{1}\left(\mathbb{R}^{d}\right) \rightarrow \mathbb{C}$ given by

$$
\Psi(\varepsilon)[u, v]:=\int_{\mathbb{R}^{d}} A\left(\frac{x}{\varepsilon}\right) \nabla_{x} u(x) \cdot \overline{\nabla_{x} v(x)}+a_{0}\left(\frac{x}{\varepsilon}\right) u(x) \overline{v(x)} d x .
$$

It was shown in (3) that there exists $\nu_{0}>0$, such that for all $0<\nu<\nu_{0}, f \in\left(H_{\nu}^{1}\left(\mathbb{R}^{d}\right)\right)^{*}$ and for all $\varepsilon>0$ there exists a unique weak solution $u^{\varepsilon}(x) \in H_{-\nu}^{1}\left(\mathbb{R}^{d}\right)$ of the variational problem

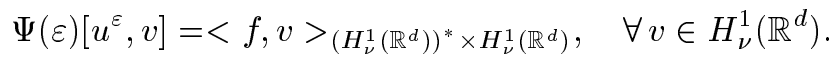

The brackets $\left\langle\cdot, \cdot>_{\left(H_{\nu}^{1}\left(\mathbb{R}^{d}\right)\right)^{*} \times H_{\nu}^{1}\left(\mathbb{R}^{d}\right)}\right.$ on the right hand side in (2.3) stand for the $\left(H_{\nu}^{1}\left(\mathbb{R}^{d}\right)\right)^{*} \times H_{\nu}^{1}\left(\mathbb{R}^{d}\right)$ duality paring and are the natural extension of the $L^{2}$ scalar product in $\mathbb{R}^{d}$ (in the sense that $H_{\nu}^{1}\left(\mathbb{R}^{d}\right) \subset$ $\left.L^{2}\left(\mathbb{R}^{d}\right) \cong L^{2}\left(\mathbb{R}^{d}\right)^{*} \subset\left(H_{\nu}^{1}\left(\mathbb{R}^{d}\right)\right)^{*}\right)$. This means that (1.2) can even be solved when $f$ belongs to a larger class of functions than $L^{2}\left(\mathbb{R}^{d}\right)$, namely to the dual space of $H_{\nu}^{1}\left(\mathbb{R}^{d}\right)$. Such functions are for example polynomials or $e^{i<t, \cdot>}$, for $t \in \mathbb{C}^{d},|\operatorname{Im} t|<\nu$. Moreover, the solution operator is continuous from $\left(H_{\nu}^{1}\left(\mathbb{R}^{d}\right)\right)^{*}$ into $H_{-\nu}^{1}\left(\mathbb{R}^{d}\right)$. 
Consider now the parameter dependent family of bounded linear functionals on $H_{\nu}^{1}\left(\mathbb{R}^{n}\right)$ given by the standard Fourier waves $e^{i<t, \cdot>} \in\left(H_{\nu}^{1}\left(\mathbb{R}^{d}\right)\right)^{*}$ parametrized with respect to $t \in \mathbb{R}^{d}$. Then, for each frequency $t \in \mathbb{R}^{d}$ we denote by $\psi(x / \varepsilon, \varepsilon, t) \in H_{-\nu}^{1}\left(\mathbb{R}^{d}\right)$ the unique weak solution of (2.3) with respect to $f(\cdot)=e^{i<t, \cdot>}$. It has been shown in (3) that $\psi(y, \varepsilon, t)=\phi(y, \varepsilon, t) e^{i \varepsilon<t, y>}$, with $\phi(y, \varepsilon, t) \in H_{p e r}^{1}(\hat{Q})$ being the solution of the so-called unit cell problem in $\hat{Q}:=(-\pi, \pi)^{d}$ : For $\varepsilon>0$ and $t \in \mathbb{C}^{d}$, find $\phi(y, \varepsilon, t) \in H_{p e r}^{1}(\hat{Q})$ such that

$$
\Phi(\varepsilon, t)[\phi, v]=\varepsilon^{2}(1, v)_{0, \hat{Q}}, \quad \forall v \in H_{p e r}^{1}(\hat{Q}) .
$$

The bilinear form $\Phi(\varepsilon, t)[\cdot, \cdot]: H_{\text {per }}^{1}(\hat{Q}) \times H_{p e r}^{1}(\hat{Q}) \rightarrow \mathbb{C}$ in $(2.4)$ is defined by

$$
\Phi(\varepsilon, t)[\phi, v]=\int_{\hat{Q}} A(y) \nabla_{y}\left(\phi(y) e^{i \varepsilon<t, y>}\right) \cdot \overline{\nabla_{y}\left(v(y) e^{i \varepsilon<t, y>}\right)}+\varepsilon^{2} a_{0}(y) \phi(y) \overline{v(y)} d y .
$$

The significance of the unit-cell problem (2.4) lies in the fact that for every $f \in L^{2}\left(\mathbb{R}^{d}\right)$, the solution $u^{\varepsilon}(x)$ of $(2.3)$ has a representation as a (generalized) Fourier-Inversion integral

$$
u^{\varepsilon}(x)=\frac{1}{(2 \pi)^{d / 2}} \int_{\mathbb{R}^{d}}^{(B)} \hat{f}(t) e^{i<t, x>} \phi\left(\frac{x}{\varepsilon}, \varepsilon, t\right) d t,
$$

with integral kernel $\psi(x / \varepsilon, \varepsilon, t)=\phi(x / \varepsilon, \varepsilon, t) e^{i<t, x>}$. The integral in (2.5) has to be understood in the sense of Bochner integral of Banach space valued function (see (3) for a definition). Henceforth, we write $\int^{(B)}$ for such integrals. The representation (2.5) will be the basis for our construction of $\left\{V_{n}\right\}_{n}$. We have, see $((7),(8))$

Proposition 2.1 There exists $\delta>0$ depending only on $A, a_{0}, \nu$, such that for any

$$
t \in \mathcal{D}_{\delta}:=\left\{t \in \mathbb{C}^{d}:|\operatorname{Im} t|<\delta\right\}
$$

and every $\varepsilon>0$, problem (2.4) admits a unique solution $\phi(\cdot, \varepsilon, t) \in H_{p e r}^{1}(\hat{Q})$. Moreover,

a) for any fixed $\varepsilon>0$, the kernel $\phi(\cdot, \varepsilon, t)$ is an analytic $H_{p e r}^{1}(\hat{Q})$-valued function of $t$ in the strip $\mathcal{D}_{\delta} \subset \mathbb{C}^{d}$,

b) for any $(\varepsilon, t)$ in $\mathbb{R}^{d+1}, \phi(\cdot, \varepsilon, t)$ is real analytic at $(\varepsilon, t)$ (with domain of analyticity depending on $(\varepsilon, t)$, however $)$.

Remark 2.2 A very useful regularity property holds for the kernel $\psi(\cdot / \varepsilon, \varepsilon, t)$. It has been shown in (8) that $\psi(\cdot / \varepsilon, \varepsilon, t)$ can be extended as analytic and uniformly bounded function of $t$ in the strip $\mathcal{D}_{\delta}$ with values in $H_{-\nu}^{1}\left(\mathbb{R}^{d}\right)$, i.e. $\psi(\cdot / \varepsilon, \varepsilon, t) \in \mathcal{A}\left(\mathcal{D}_{\delta}, H_{-\nu}^{1}\left(\mathbb{R}^{d}\right)\right) \cap L^{\infty}\left(\mathcal{D}_{\delta}, H_{-\nu}^{1}\left(\mathbb{R}^{d}\right)\right)$, with bounds independent of $\varepsilon>0$.

\section{$2.2 \quad$ Approximation on $\mathbb{R}^{d}$}

We return to the question raised in Section 1, i.e. if subspace sequences $\left\{V_{n}\right\}_{n}$ (possibly depending on $\varepsilon$ ) can be found which realize the convergence rates (1.7). One approach would be to incorporate analytic results from classical homogenization, i.e. an asymptotic expansion of $u^{\varepsilon}(x)$ as $\varepsilon \rightarrow 0((5)$, (6)), into the FEM. This "classical homogenization approach" can also be derived from (2.5) (see (3), (8)), but does not give subspaces $V_{n}$ which achieve (1.7) (7). To do so, we must exploit the analytic 
structure of the data $f$ and of the kernel $\psi(x / \varepsilon, \varepsilon, t)$ in (2.5) indicated in Remark 2.2.

The idea is to approximate the Fourier-Bochner integral (2.5) by a finite sum, which is obtained by truncating a (generalized) Poisson summation formula.

For $N \in \mathbb{N}$ and $h>0$ define the trapezoidal approximation of (2.5)

$$
u_{N, h}^{\varepsilon}(x)=\mathbf{1}_{\left[-\frac{\pi}{h}, \frac{\pi}{h}\right]^{d}}(x) \frac{1}{(2 \pi)^{d / 2}} h^{d} \sum_{k \in \mathbb{Z}_{(N)}^{d}} \hat{f}(k h) \phi\left(\frac{x}{\varepsilon}, \varepsilon, k h\right) e^{i<k h, x>},
$$

where

$$
\mathbb{Z}_{(N)}^{d}=\left\{k \in \mathbb{Z}^{d}:\left|k_{j}\right| \leq N, \forall j=1, \ldots, d\right\}
$$

Definition 2.3 We say a function $f$ fulfills the 'usual assumptions' if $f \in L^{2}\left(\mathbb{R}^{d}\right)$, and its Fourier transformation $\hat{f}(\cdot)$ can be extended to a holomorphic function in the strip $\mathcal{D}_{\delta}$, with $\delta$ as in Proposition 2.1, which satisfies the following growth condition:

$$
|\hat{f}(z)| \leq C_{f} e^{-\alpha|z|}, \quad \forall z \in \mathcal{D}_{\delta}
$$

for some positive constants $C_{f}, \alpha>0$.

Our main result on the trapezoidal approximation (2.6) of the Fourier-Bochner integral (2.5) is (see (8) for a proof) :

Proposition 2.4 Let us assume that $f$ satisfies the 'usual assumptions' as in Definition 2.3, L $>0$ is arbitrary and the step size $h$ is given by

$$
h=\left(\frac{\pi \delta}{\alpha N}\right)^{1 / 2}, \quad N \geq \frac{4 \delta L^{2}}{\alpha \pi} .
$$

Then the error $\delta_{N}(f, h)(\cdot):=u^{\varepsilon}(\cdot)-u_{N, h}^{\varepsilon}(\cdot)$, with $u_{N, h}^{\varepsilon}(\cdot)$ as in (2.6), decays exponentially with respect to $N$ in the $\|\cdot\|_{0,-2 \nu},\|\cdot\|_{H_{-2 \nu}^{1}}\left((-L, L)^{d}\right)^{-n o r m s:}$

$$
\left\|\delta_{N}(f, h)(\cdot)\right\|_{0,-2 \nu}+\left\|\delta_{N}(f, h)(\cdot)\right\|_{H_{-2 \nu}^{1}\left((-L, L)^{d}\right)} \leq C_{0} C_{f} \frac{1}{\alpha^{d}} e^{-(\pi \alpha \delta N / d)^{1 / 2}}
$$

with the constant $C_{0}=C(\gamma, \nu, d, \alpha)$ independent of $\varepsilon, N$ and $L$.

Remark 2.5 Let $\mathcal{V}_{\varepsilon}^{N}:=\operatorname{Span}\left\{\operatorname{Re} \psi(\dot{\varepsilon}, \varepsilon, k h), \operatorname{Im} \psi(\dot{\varepsilon}, \varepsilon, k h): k \in \mathbb{Z}_{(N)}^{d}\right\}$. Then $n=\operatorname{dim} \mathcal{V}_{\varepsilon}^{N}=$ $O\left(N^{d}\right)$, and we see from (2.9) that $u^{\varepsilon}$ is approximated by $\mathcal{V}_{\varepsilon}^{N}$ at a robust (in $\varepsilon$ ) exponential rate, but comparing with (1.7) the approximation is not optimal in the sense of $n$-width.

\subsection{Periodic setting}

We will now exhibit an example of subspaces where the optimal bound (1.7) can be achieved. In order to present the ideas clearly, we consider the bounded domain in the periodic setting (non-periodic below). Let $1 / \varepsilon \in \mathbb{N}$ and $\Omega=(0,2 \pi)^{d}$ be an $1 / \varepsilon$ fold repetition of the scaled unit cell $Q=\varepsilon \hat{Q}$ :

$$
\bar{\Omega}=\bigcup_{k \in \mathbb{Z}^{d}: 0 \leq k_{i}<1 / \varepsilon} \varepsilon((2 k+1) \pi+\overline{\hat{Q}}) .
$$


Likewise $A(y)=A(y+2 k \pi), a_{0}(y)=a_{0}(y+2 k \pi), \forall k \in \mathbb{Z}^{d}$ and assume that $f(x)=f(x+2 k \pi) \in L^{2}(\Omega)$. Denote by $u^{\varepsilon}$ the solution of the following problem

$$
u^{\varepsilon} \in H_{p e r}^{1}(\Omega): L^{\varepsilon} u^{\varepsilon}=f \quad \text { in } \Omega,
$$

where $L^{\varepsilon}$ is the second order, strongly elliptic operator

$$
L^{\varepsilon} u=-\nabla \cdot\left(A\left(\frac{x}{\varepsilon}\right) \nabla u\right)+a_{0}\left(\frac{x}{\varepsilon}\right) u .
$$

Discrete analog of (2.5). Let us denote by $f_{\tau}$ the Fourier coefficients of $f$ with respect to the basis $\left\{e^{i<\tau, x>}\right\}_{\tau \in \mathbb{Z}^{d}} \subset L^{2}(\Omega)$ :

$$
f(x)=\sum_{\tau \in \mathbb{Z}^{d}} f_{\tau} e^{i<\tau, x>} .
$$

Then, it can be easily seen that

$$
u^{\varepsilon}(x)=\sum_{\tau \in \mathbb{Z}^{d}} f_{\tau} \phi\left(\frac{x}{\varepsilon}, \varepsilon, \tau\right) e^{i<\tau, x>}=\sum_{\tau \in \mathbb{Z}^{d}} f_{\tau} \psi\left(\frac{x}{\varepsilon}, \varepsilon, \tau\right),
$$

where $\phi(\cdot, \varepsilon, t)$ is as in (2.4) and the series (2.12) converges in the Banach space $H_{p e r}^{1}(\Omega)$.

Define for $\mu \in \mathbb{N}$ and $\varepsilon>0$

$$
\tilde{\mathcal{V}}_{\varepsilon}^{\mu}:=\operatorname{Span}\left\{\operatorname{Re} \psi\left(\frac{\dot{\varepsilon}}{\varepsilon}, \varepsilon, \tau\right), \operatorname{Im} \psi\left(\frac{\dot{\varepsilon}}{\varepsilon}, \varepsilon, \tau\right), \tau \in \mathbb{Z}^{d}, 0 \leq|\tau| \leq \mu\right\} .
$$

Then $n=\operatorname{dim}\left(\tilde{\mathcal{V}}_{\varepsilon}^{\mu}\right)=O\left(\mu^{d}\right)$ and the following approximation result holds

Proposition 2.6 Assume that $f \in \mathcal{A}_{\text {per }}(\bar{\Omega})$. Then, for every $\varepsilon>0$ such that $1 / \varepsilon \in \mathbb{N}$,

$$
\inf _{v \in \tilde{\mathcal{V}}_{\varepsilon}^{\mu}}\left\|u^{\varepsilon}-v\right\|_{1, \Omega} \leq C e^{-b \mu}=C \exp \left(-b n^{1 / d}\right),
$$

where $C, b>0$ are independent of $\varepsilon, \mu$, depend only on $f$.

We see that the subspace sequence $\left\{V_{n}\right\}_{n}$ given by $\left\{\tilde{\mathcal{V}}_{\varepsilon}^{\mu}\right\}_{\mu}$ in $(2.13)$ (with $n=O\left(\mu^{d}\right)$ ) realizes the optimal rate in Theorem 1.1.

\section{Generalized $p$-FEM in homogenization}

So far, Propositions 2.4, 2.6 indicated that for the model problem

$$
L^{\varepsilon} u^{\varepsilon}:=-\nabla \cdot\left(A\left(\frac{x}{\varepsilon}\right) \nabla u^{\varepsilon}(x)\right)+a_{0}\left(\frac{x}{\varepsilon}\right) u^{\varepsilon}(x)=f(x)
$$

in either the unbounded domain or the periodic setting, with analytic $f$, the solution $u^{\varepsilon}$ can be approximated very well by linear combinations of the kernel $\psi(x / \varepsilon, \varepsilon, t)\left(\operatorname{resp} . \phi(x / \varepsilon, \varepsilon, t) e^{i<t, x>}\right)$.

In a general, bounded domain $\Omega \subset \mathbb{R}^{d}$ we propose to construct therefore generalized FE-spaces $S^{p, \mu}(\Omega, \mathcal{T})$ which consist of the "usual" piecewise polynomial functions of degree $p$ which are augmented by special, so-called 'micro' shape functions of 'degree' $\mu$ derived from the sampled kernel $\phi$. Clearly, these shape functions are generally not explicitly known and must be computed numerically by a FE-solution of the unit-cell problem. 


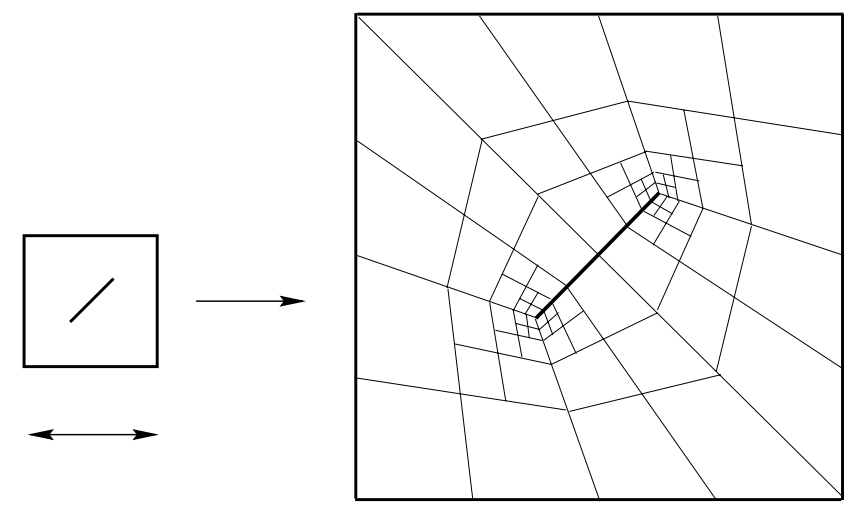

Figure 2: Geometric mesh $\hat{\mathcal{T}}$ for unit cell $\hat{Q}$ with crack.

\subsection{Computation of microscale shape functions.}

Let $\varepsilon>0, t \in \mathbb{C}^{d}$ be given, and $\hat{\mathcal{T}}$ an affine mesh on the unit cell $\hat{Q}$. Then the FE formulation for the unit cell-problem $(2.4)$ reads: Find $\phi_{h p}(\cdot, \varepsilon, t) \in S_{p e r}^{\hat{p}, 1}(\hat{Q}, \hat{\mathcal{T}})$, such that

$$
\Phi(\varepsilon, t)\left[\phi_{h p}, v\right]=\varepsilon^{2}(1, v)_{0, \hat{Q}}, \quad \forall v \in S_{p e r}^{\hat{p}, 1}(\hat{Q}, \hat{\mathcal{T}}),
$$

where the FE space $S_{p e r}^{\hat{p}, 1}(\hat{Q}, \hat{\mathcal{T}})$ is defined by

$$
S_{p e r}^{\hat{p}, 1}(\hat{Q}, \hat{\mathcal{T}}):=\left\{u \in H_{p e r}^{1}(\hat{Q}):\left.u\right|_{K} \in \mathcal{S}^{\hat{p}}(K), \forall K \in \hat{\mathcal{T}}\right\}
$$

and $\mathcal{S}^{\hat{p}}(K)$ is the space of polynomials of degree at most $\hat{p}$ on $K$. The design of $\hat{\mathcal{T}}, \hat{p}$ must take into account regularity of the solution, e.g. if the unit cell problem has a crack, then the solution $\phi(y, \varepsilon, t) \in B_{\beta}^{2}(\hat{Q})$, the countably normed space (1), and $h p$-FEM with a geometrical mesh refinement towards the crack-tips must be employed for the solution of the unit-cell problem. (see Fig. 2).

The kernel $\phi(\cdot / \varepsilon, \varepsilon, t)$ can be directly employed for computational purposes, and we see from (2.9), (2.14) that collocation of $\phi(\cdot / \varepsilon, \varepsilon, t)$ at various sets of collocation points $\mathcal{N}=\left\{t_{j}\right\}$ gives systems of shape functions with very good approximation properties for elliptic problems with periodic microstructure.

Since $\phi(y, \varepsilon, t)$ is analytic in $t$ (Proposition 2.1), choosing the collocation points $\mathcal{N}=\left\{k h, k \in \mathbb{Z}_{(N)}^{d}\right\}$ with $h \rightarrow 0$ as in (2.8) will lead to almost linear dependence of $\phi_{h p}\left(y, \varepsilon, t_{j}\right), t_{j} \in \mathcal{N}$; these functions are therefore unsuitable as basis for $p$-FEM in numerical computations. We propose here a methodology to derive a well conditioned set of micro shape functions from the collocated kernels $\psi\left(\cdot / \varepsilon, \varepsilon, t_{j}\right)$, which is based on 'oversampling $+S V D$ '. As a byproduct, this approach will also reduce the number of micro shape functions substantially. To this end, we select the collocation points

$$
\mathcal{N}:=\left\{t_{j}: j=1, \ldots, \hat{\mu}\right\}, \quad \hat{\mu}>\mu
$$

and orthogonalize by SVD the matrix of coefficient vectors of the FE approximations to the $\phi\left(y, \varepsilon, t_{j}\right), t_{j} \in$ $\mathcal{N}$. Select therefore $\underline{N}(y)$ to be a well conditioned basis of $S_{p e r}^{\hat{p}, 1}(\hat{Q}, \hat{\mathcal{T}})$ and denote by $\Phi_{j}(\varepsilon)$ the coefficient vector of $\phi_{h p}\left(y, \varepsilon, t_{j}\right)$ with respect to $\underline{N}(y)$, i.e. $\phi_{h p}\left(y, \varepsilon, t_{j}\right)=\Phi_{j}(\varepsilon)^{\top} \underline{N}(y)$. Compute the SVD of the matrix of coefficient vectors $\Phi_{j}(\varepsilon), j=1, \ldots, \hat{\mu}$

$$
\left[\Phi_{1}(\varepsilon), \ldots, \Phi_{\hat{\mu}}(\varepsilon)\right]=\underline{U} \operatorname{diag}\left(\sigma_{1}, \ldots, \sigma_{\hat{\mu}}\right) \underline{V}^{\top}
$$


and define the basis functions

$$
\tilde{\phi}_{j}\left(\frac{x}{\varepsilon}, \varepsilon\right):=\underline{U}_{j}^{\top} \underline{N}\left(\frac{x}{\varepsilon}\right), j=1, \ldots, \hat{\mu}
$$

with $\underline{U}_{j}$ being the $j$-th column of $\underline{U}$.

Note that $\operatorname{Span}\left\{\tilde{\phi}_{j}(y, \varepsilon): j=1, \ldots, \hat{\mu}\right\}=\operatorname{Span}\left\{\phi_{h p}\left(y, \varepsilon, t_{j}\right): t_{j} \in \mathcal{N}\right\}$. Define further the micro space

$$
\mathcal{M}_{\varepsilon}^{\mu}=\operatorname{Span}\left\{\tilde{\phi}_{j}\left(\frac{x}{\varepsilon}, \varepsilon\right): j=1, \ldots, \mu+1\right\},
$$

where $\mu=0, \ldots, \mu^{*}, \mu^{*}=\inf \left\{j: \sigma_{j}>t o l\right\}$ and $t o l$ is a parameter of order of machine precision. We present in Fig. 3 the shape functions $\left\{\tilde{\phi}_{j}(y, \varepsilon)\right\}_{j=0}^{\mu}$ obtained by the above procedure, in the one dimensional case, for $\varepsilon=0.001, a_{0} \equiv 1$ and

$$
A(y)= \begin{cases}10, & \text { if }|y| \leq \frac{\pi}{2} \\ 1, & \text { else }\end{cases}
$$

In this case, we selected the first $\mu+1=5$ shape functions $\tilde{\phi}_{j}(y, \varepsilon)$ corresponding to singular values $\sigma_{j}>10^{-10}$.

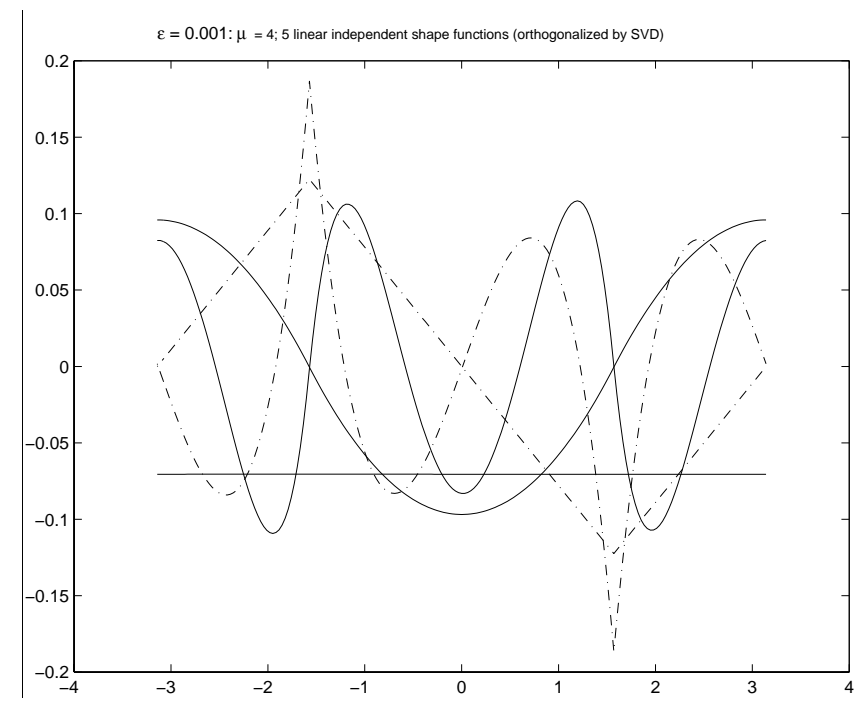

Figure 3: $\tilde{\phi}_{j}(\cdot, \varepsilon), j=0, \ldots, \mu=4$.

Remark 3.1 We see in Fig. 3 that $\tilde{\phi}_{1}(y, \varepsilon) \equiv$ const.

Remark 3.2 In numerical experiments we found this algorithm very robust with respect to the choice of collocation points. After the SVD the first shape functions associated with the largest singular values are practically independent of the number and of the choice of $t_{j}$. The resulting micro shape functions $\tilde{\phi}_{j}(\cdot, \varepsilon)$ are therefore, at least numerically, hierarchical, and enable hierarchic modeling of problems with microstructure. 


\subsection{Construction of generalized FE spaces.}

Let $\Omega \subset \mathbb{R}^{d}$ be an open, bounded polygonal domain and assume that $f \in L^{2}(\Omega)$. We denote by $u^{\varepsilon}$ the unique weak solution of the following boundary value problem: find $u^{\varepsilon} \in H_{0}^{1}(\Omega)$, such that

$$
\int_{\Omega} A\left(\frac{x}{\varepsilon}\right) \nabla u^{\varepsilon} \cdot \nabla v+a_{0}\left(\frac{x}{\varepsilon}\right) u^{\varepsilon} v d x=(f, v)_{0, \Omega}, \quad \forall v \in H_{0}^{1}(\Omega) .
$$

It is well known (5), (6) that in homogenization on bounded domains there arise boundary correctors that are not accounted for in (2.5). In the design of $S \underline{p}, \underline{\mu}(\Omega, \mathcal{T})$ we need to account for them. We deal with this by selecting the (macro) mesh $\mathcal{T}$ of the generalized FEM properly. To motivate this selection, let

$$
\begin{gathered}
\tilde{\mathcal{V}}_{\varepsilon}^{\mu}:=\operatorname{Span} \quad\left\{\begin{array}{l}
\operatorname{Re} D_{t}^{\alpha} \psi\left(\frac{\dot{-}}{\varepsilon}, \varepsilon, 0\right), \alpha \in \mathbb{N}^{d},|\alpha|=2 k,|\alpha| \leq \mu, \\
\left.\operatorname{Im} D_{t}^{\alpha} \psi\left(\frac{\dot{g}}{\varepsilon}, \varepsilon, 0\right),|\alpha|=2 k+1,|\alpha| \leq \mu\right\},
\end{array}\right. \\
\hat{\mathcal{V}}_{\varepsilon}^{\mu}:=\operatorname{Span} \quad\left\{\begin{array}{l}
\operatorname{Re} v_{\alpha}^{\varepsilon}, \alpha \in \mathbb{N}^{d},|\alpha|=2 k,|\alpha| \leq \mu, \\
\left.\operatorname{Im} v_{\alpha}^{\varepsilon},|\alpha|=2 k+1,|\alpha| \leq \mu\right\},
\end{array}\right.
\end{gathered}
$$

where by $D_{t}^{\alpha}$ we denote the partial derivative of order $\alpha$ with respect to $t: D_{t}^{\alpha}:=\partial_{t_{1}}^{\alpha_{1}} \partial_{t_{2}}^{\alpha_{2}} \ldots \partial_{t_{d}}^{\alpha_{d}}$, $|\alpha|=\alpha_{1}+\ldots+\alpha_{d}$ and $v_{\alpha}^{\varepsilon} \in H^{1}(\Omega)$ is the solution of the following boundary value problem with homogeneous right hand side and inhomogeneous boundary data :

$$
\begin{aligned}
-\nabla \cdot\left(A\left(\frac{x}{\varepsilon}\right) \nabla v_{\alpha}^{\varepsilon}\right)+a_{0}\left(\frac{x}{\varepsilon}\right) v_{\alpha}^{\varepsilon} & =0 \text { in } \Omega \\
\left.v_{\alpha}^{\varepsilon}\right|_{\partial \Omega} & =-\left.D_{t}^{\alpha} \psi\left(\frac{\dot{\varepsilon}}{\varepsilon}, \varepsilon, 0\right)\right|_{\partial \Omega}, \quad \alpha \in \mathbb{N}^{d} .
\end{aligned}
$$

Set

$$
\mathcal{V}_{\varepsilon}^{\mu}=\left(\tilde{\mathcal{V}}_{\varepsilon}^{\mu}+\hat{\mathcal{V}}_{\varepsilon}^{\mu}\right) \bigcap H_{0}^{1}(\Omega)
$$

Then the following result holds $(8 ; 7)$ :

Proposition 3.3 Assume that $f \in \mathcal{A}(\bar{\Omega})$ and let $u^{\varepsilon}$ be the solution of (3.8). Then, there exist positive constants depending on $f$, but independent of $\varepsilon$ and $\mu$, such that

$$
\inf _{v \in \mathcal{V}_{\varepsilon}^{\mu}}\left\|u^{\varepsilon}-v\right\|_{1, \Omega} \leq C e^{-b \mu}
$$

This indicates that $\psi(\cdot / \varepsilon, \varepsilon, t)$ for small $t$ are most important for problems with smooth data $f$, since $D_{t}^{\alpha} \psi(\cdot / \varepsilon, \varepsilon, 0)$ solves $(2.2)$ with $f=(i x)^{\alpha}$; the space $\hat{\mathcal{V}}_{\varepsilon}^{\mu}$ is needed here to enforce the boundary conditions. We observe that $\tilde{\mathcal{V}}_{\varepsilon}^{\mu}$ is spanned by products of 'micro' shape functions $D_{t}^{\alpha} \phi(\cdot / \varepsilon, \varepsilon, 0)$ times polynomials of degree at most $\mu$. The correctors $v_{\alpha}^{\varepsilon}$ in (3.11) are solutions of the homogeneous equation $L^{\varepsilon} u^{\varepsilon}=0$ in $\Omega$ with inhomogeneous boundary data $(5 ; 6)$. The spaces $S^{p} \otimes \mathcal{M}_{\varepsilon}^{\mu}$ approximate solutions of $L^{\varepsilon} u^{\varepsilon}=0$ locally at a spectral rate (see (7) or (8) Proposition 3.12).

Of course, in the $O(\varepsilon)$ vicinity of $\partial \Omega$, the structure of the solution is not anymore well described by the representation (2.5); hence there the subspaces $\mathcal{M}_{\varepsilon}^{\mu}$ in (3.6) are expected to have poor approximation 


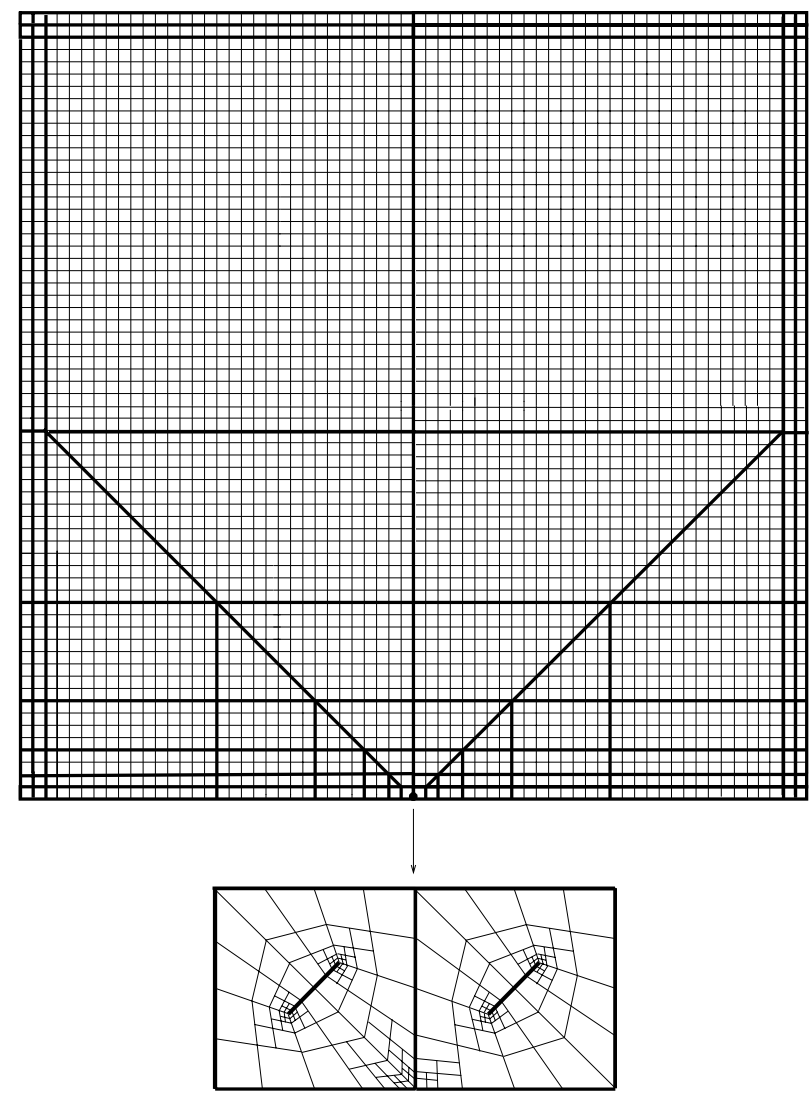

Figure 4: Macro mesh $\mathcal{T}$ (bold lines) for cracked panel with crack in each micro-cell (thin lines). Geometric mesh refinement towards 'macro' singularity at $\bullet$.

properties. Accordingly, we compose the finite element mesh $\mathcal{T}=\mathcal{T}_{\text {int }} \cup \mathcal{T}_{b} \cup \mathcal{T}_{\text {sing }}$ of three parts : a quasiuniform 'interior' part $\left(\mathcal{T}_{\text {int }}\right)$ and a refined 'boundary' part consisting of elements abutting at a corner or at a singularity $\left(\mathcal{T}_{\text {sing }}\right)$ or elongated elements abutting at the boundary $\left(\mathcal{T}_{b}\right)$, see Fig. 4 for an example. Note that in $\mathcal{T}_{\text {int }}$ the element size is independent of $\varepsilon$.

Denote by $\underline{p}=\left\{p_{K}\right\}_{K \in \mathcal{T}}$ a degree vector and define the finite element space

$$
S \underline{p}(\Omega, \mathcal{T})=\left\{u \in H^{1}(\Omega):\left.u\right|_{K} \in \mathcal{S}^{p_{K}}(K), \forall K \in \mathcal{T}\right\},
$$

where $\mathcal{S}^{p_{K}}(K)$ is elemental polynomial space. Define the micro degree vector $\underline{\mu}=\left\{\mu_{K}\right\}_{K \in \mathcal{T}}$. Then let us introduce the generalized FE-space

$$
S \underline{p}, \underline{\mu}(\Omega, \mathcal{T})=\left\{u \in H^{1}(\Omega):\left.u\right|_{K} \in \mathcal{S}^{p_{K}}(K) \otimes \mathcal{M}_{\varepsilon}^{\mu_{K}}, \forall K \in \mathcal{T}\right\}
$$


and set $S_{0}^{\underline{p}, \underline{\mu}}(\Omega, \mathcal{T})=S \underline{p}, \underline{\mu}(\Omega, \mathcal{T}) \cap H_{0}^{1}(\Omega)$.

Strictly speaking, $S \underline{\underline{p}}, \underline{\mu}(\Omega, \mathcal{T})$ also depends on $\hat{\mathcal{T}}, \hat{p}$ used in (3.3) and on the set $\mathcal{N}$ of collocation points. However, if $\hat{\mathcal{T}}$ is sufficiently fine and $\hat{p}$ in (3.3) sufficiently large, the $\phi_{h p}$ will converge quasioptimally and $\mathcal{M}_{\varepsilon}^{\mu}$ will practically not depend on $\hat{\mathcal{T}}, \hat{p}$. We therefore omit $\hat{\mathcal{T}}, \hat{p}$ in (3.14). Likewise, the collocation set $\mathcal{N}$ is not important (see Remark 3.2) and is also omitted. The vector $\underline{p}=\left\{p_{K}\right\}_{K \in \mathcal{T}}$ we call the 'macro' polynomial degree, whereas $\underline{\mu}=\left\{\mu_{K}\right\}_{K \in \mathcal{T}}$ is called the 'micro' degree of the generalized $p$-FEM. Therefore, the generalized FE space $S \underline{p}, \underline{\mu}(\Omega, \mathcal{T})$ in (3.14) accounts for both scales, 'micro' and 'macro'. The 'micro' degree vector $\underline{\mu}$ is variable; in elements near corner singularities we omit generalized shape functions :

$$
\left\{\begin{array}{cl}
\mu_{K} \cong p_{K} \quad, \quad \text { if } K \in \mathcal{T}_{\text {int }} \cup \mathcal{T}_{b}, \\
0, \quad \text { if } K \in \mathcal{T}_{\text {sing }} .
\end{array}\right.
$$

Note that in the elongated elements near the boundary, but not abutting corners, also $\mu_{K} \cong p_{K}$. This is necessary to resolve the tangential fine structure of $u^{\varepsilon}$. Note further that since $\mathcal{M}_{\varepsilon}^{0}=\operatorname{Span}\{1\}$ if $a_{0} \equiv$ const (see Remark 3.1), we get that

$$
S^{\underline{p}}(\Omega, \mathcal{T}) \subset S^{p} \underline{\underline{\mu}}(\Omega, \mathcal{T}),
$$

i.e. we have a generalized $p$-FEM. Moreover, (3.15) ensures that the spaces $S \underline{p}, \underline{\mu}(\Omega, \mathcal{T})$ are dense in $L^{2}(\Omega)$, as $p_{K} \rightarrow \infty$. The generalized FE formulation finally reads: find $u_{F E}^{\varepsilon} \in S_{0}^{\underline{p}}, \underline{\mu}(\Omega, \mathcal{T})$, such that

$$
\int_{\Omega} A\left(\frac{x}{\varepsilon}\right) \nabla u^{\varepsilon} \cdot \nabla v+a_{0}\left(\frac{x}{\varepsilon}\right) u^{\varepsilon} v d x=(f, v)_{0, \Omega}, \quad \forall v \in S_{\overline{0}}^{\underline{p}} \underline{\underline{\mu}}(\Omega, \mathcal{T}) .
$$

\section{Numerical Results.}

\subsection{Set up and basic results}

We present here preliminary numerical results obtained with the generalized FEM. The goal of the experiments is to validate our approach and to get insight on the practical selection of $p_{K}$ and $\mu_{K}$, as well as on the mesh design. We restrict our numerical examples to problem (3.8) in one dimension and show the performance of the generalized $p$-FEM with $A(\cdot)$ as in $(3.7)$ and $a_{0} \equiv 0$, on a fixed mesh $\mathcal{T}=\mathcal{T}_{\text {int }} \cup \mathcal{T}_{b}$ with $\mathcal{T}_{b}$ covering 4 periods of length $2 \pi \varepsilon$ at each boundary point and only one element $K \in \mathcal{T}_{\text {int }}$. Note that in one dimension $\mathcal{T}_{\text {sing }}=\emptyset$. As right hand side we choose $f(x)=\exp (x)$; the exact solution is in this case piecewise analytic, but non-polynomial on the microscale.

As 'micro' shape functions we employ $\left\{\tilde{\phi}_{\mu}(y, \varepsilon)\right\}_{\mu}$ obtained as in (3.4)-(3.7) by solving the unit cell problem (2.4) with $a_{0} \equiv 1$, however. In agreement with Proposition 2.4 we choose the set of collocation points $\mathcal{N}=\left\{t_{j}(\hat{\mu})=j / \sqrt{\hat{\mu}}: j=1, \ldots, \hat{\mu}\right\}$ corresponding to $\hat{\mu}=64$.

Note that computation of the stiffness matrix in (3.16) needs only a fixed number of operations, independent of $\varepsilon$. This is due to the periodicity of the coefficients $A(\cdot), a_{0}(\cdot)$ and of the 'micro' shape functions $\tilde{\phi}_{\mu}(\cdot, \varepsilon)$. In fact, integrals of products of micro shape functions $\tilde{\phi}_{\mu}(y, \varepsilon) \tilde{\phi}_{\mu^{\prime}}(y, \varepsilon)$ times monomials up to degree $2 p_{K}$ over the unit cell $\hat{Q}$ have to be computed only once.

The goal of the numerical experiments is to show robust (with respect to $\varepsilon$ ) exponential convergence. In Fig. 5 we present the relative error of the energy versus 'macro' polynomial degree $p_{K}=p, \forall K \in \mathcal{T}$, by increasing 'micro' degree $\mu:=\mu_{K}, K \in \mathcal{T}_{\text {int }}$, and for different $\varepsilon$ scales varying from $\cong 10^{-6}$ up to $\cong 10^{-1}$. We see that taking $\mu=0$, which corresponds to the case when only macroscopic shape functions are used, we do not achieve convergence (only in the case $\varepsilon \cong 10^{-1}$ a very slow convergence 

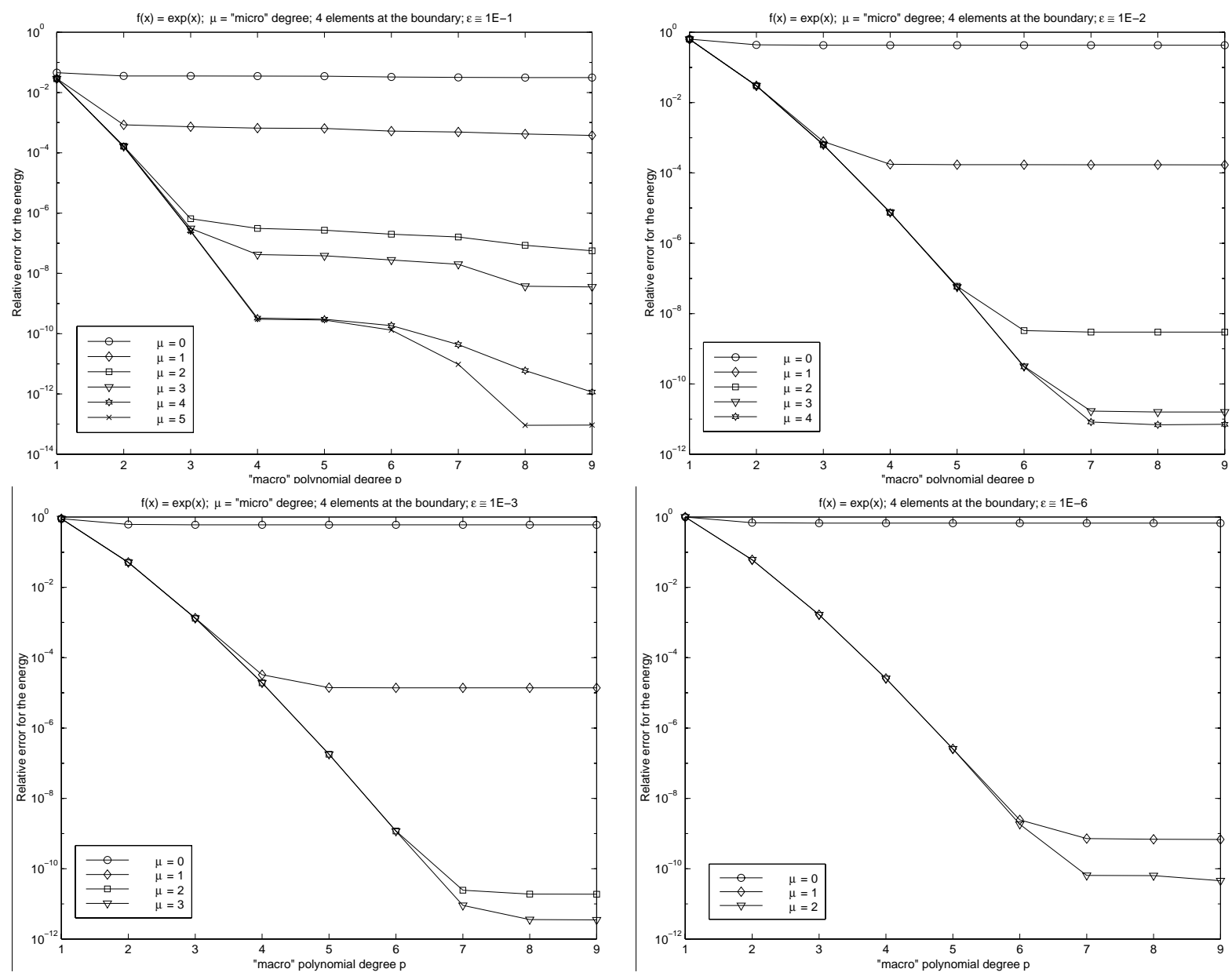

Figure 5: Exponential rate of convergence for the FE energy. $f(x)=e^{x}$

hardly visible in Figure 5 occurs, since here the scales are resolved, however the low solution regularity stalls the spectral convergence). For $\mu>0$ and increasing $p$ we get exponential convergence, however a saturation occurs at levels depending only on $\mu$. To achieve robust exponential convergence one has to augment simultaneously the standard polynomial spaces and the number of microscale shape functions.

Fig. 6 shows exponential convergence with respect to the (stronger) $W^{1, \infty}(\Omega)$ - norm, namely that the convergence rate for the stresses

$$
\left\|A\left(\frac{x}{\varepsilon}\right) \frac{d}{d x}\left(u^{\varepsilon}-u_{F E}^{\varepsilon}\right)\right\|_{L^{\infty}(-1,1)}
$$

is also exponential and independent of $\varepsilon$. 

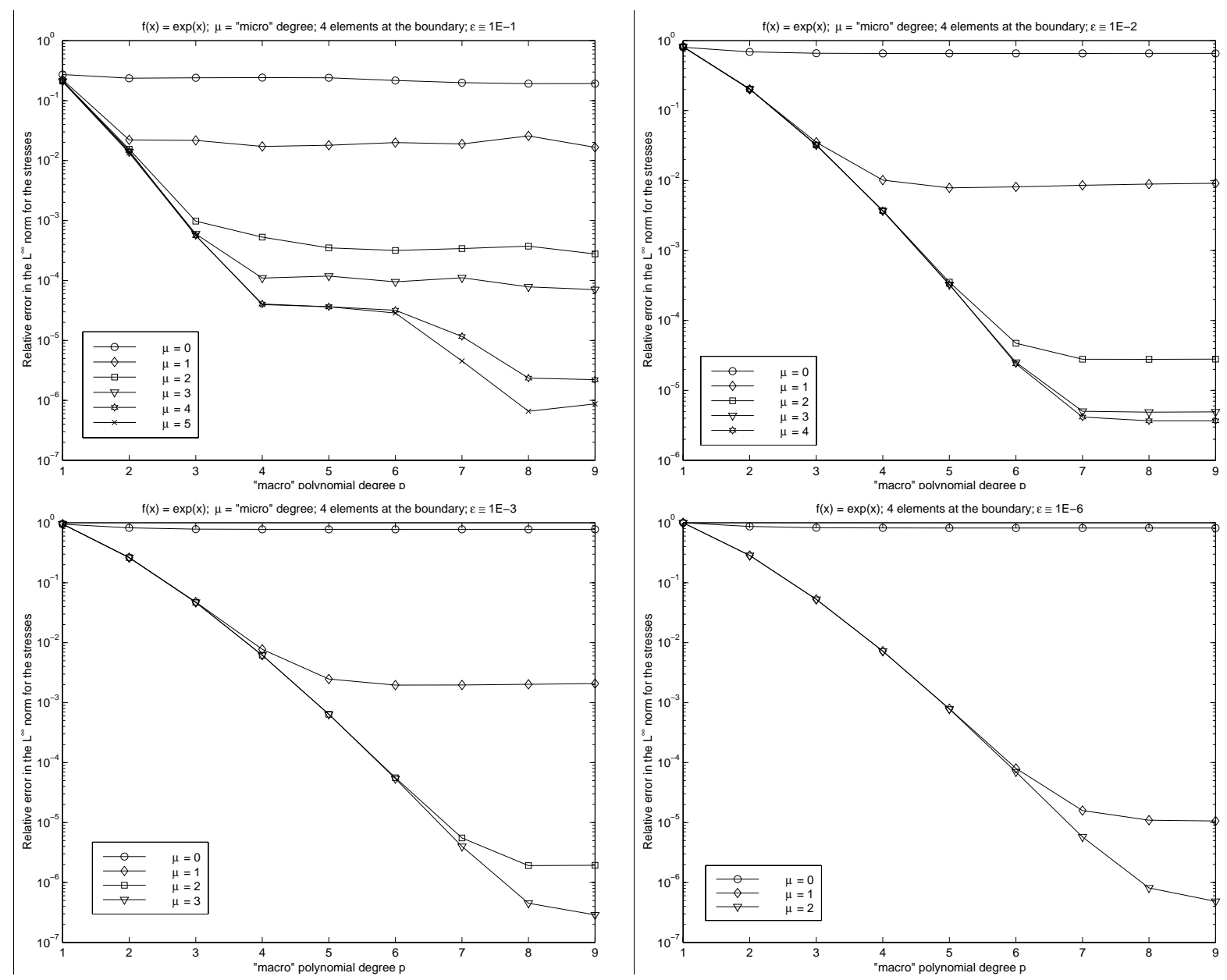

Figure 6: Exponential rate of convergence in the $L^{\infty}$ norm for the stresses. $f(x)=e^{x}$

\subsection{Jumps in coefficients}

Next, we consider the performance of our approach if the coefficient $A(\cdot)$ has a very large jump $\delta^{-1}=$ $A_{\text {max }} / A_{\text {min }}$, where $A_{\text {max }}:=\max _{y \in \hat{Q}} A(y)$ and $A_{\text {min }}:=\min _{y \in \hat{Q}} A(y)$

$$
A(y)=\left\{\begin{array}{cl}
\delta^{-1}, & \text { if }|y| \leq \frac{\pi}{2} \\
1, & \text { else }
\end{array}\right.
$$

As $\delta \rightarrow 0$, we found the generalized $p$-FEM to be stable. Comparing Fig. 7 and Fig. 5 (for $\delta=0.1$ ) we see that the convergence is insensitive to decreasing $\delta$, the error decay is practically the same for $\delta=0.1,0.01,0.001$, but at fixed $\mu$ the error saturation occurs somewhat earlier as $\delta \rightarrow 0$. 

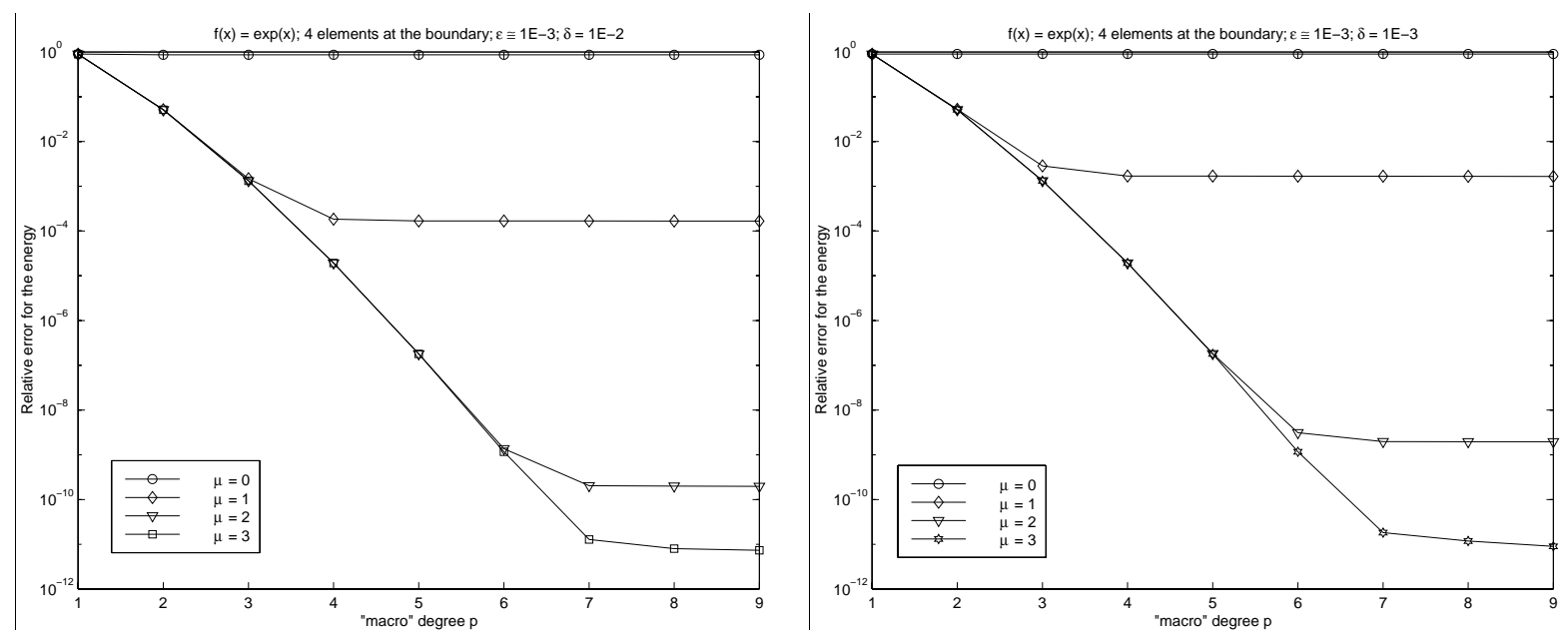

Figure 7: Exponential rate of convergence for large coefficient jumps
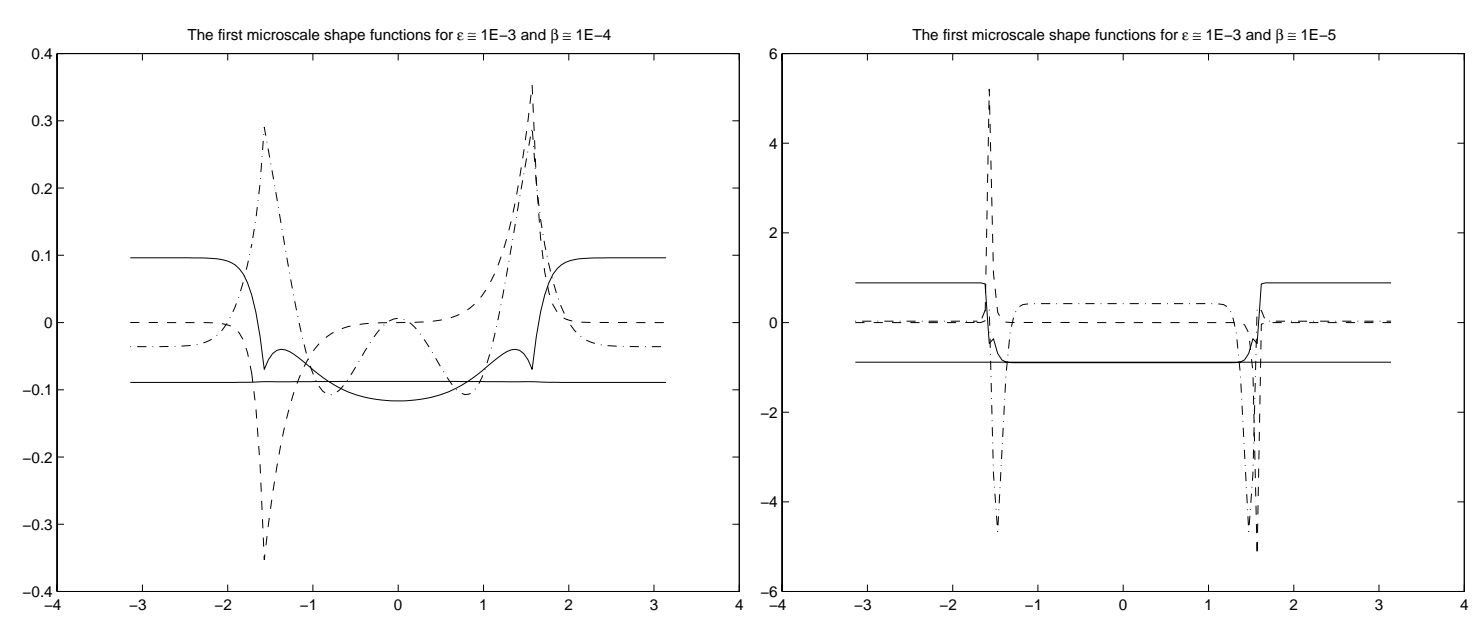

Figure 8: The microscale shape functions $\left\{\tilde{\phi}_{\mu}(y, \varepsilon)\right\}_{\mu=0, \ldots, 3}$ for $\varepsilon \approx 10^{-3}$ and $\beta \approx 10^{-4}, \beta \approx 10^{-5}$ respectively

\subsection{Singular perturbation and homogenization}

Finally, we investigate the performance of our approach if the differential operator $L^{\varepsilon}=L_{\beta}^{\varepsilon}$ in (3.1) is singularly perturbed, in the sense that the principal part depends additionally on another small parameter $0<\beta<<1$ in the following way

$$
L_{\beta}^{\varepsilon} u_{\beta}^{\varepsilon}=-\beta^{2} \nabla \cdot\left(A\left(\frac{x}{\varepsilon}\right) \nabla u_{\beta}^{\varepsilon}(x)\right)+a_{0}\left(\frac{x}{\varepsilon}\right) u_{\beta}^{\varepsilon}(x) .
$$

We have a problem with multiple scales : in the vicinity of each discontinuity of $A(x / \varepsilon)$, a boundary layer of thickness $\beta$ appears for $\beta<\varepsilon$. A conventional FEM would thus be required to resolve the smallest scale $\beta$. In our approach, the singular perturbation is taken care of by the unit-cell problem (2.4), which is, for $\beta / \varepsilon<<1$, itself singularly perturbed. Fig. 8 shows the $\left\{\tilde{\phi}_{\mu}(y, \varepsilon)\right\}_{\mu=0, \ldots, 3}$ for $(4.2)$ 
and for $a_{0} \equiv 1, A(\cdot)$ as in (3.7). The layers of thickness $O(\beta / \varepsilon)$ are clearly visible.
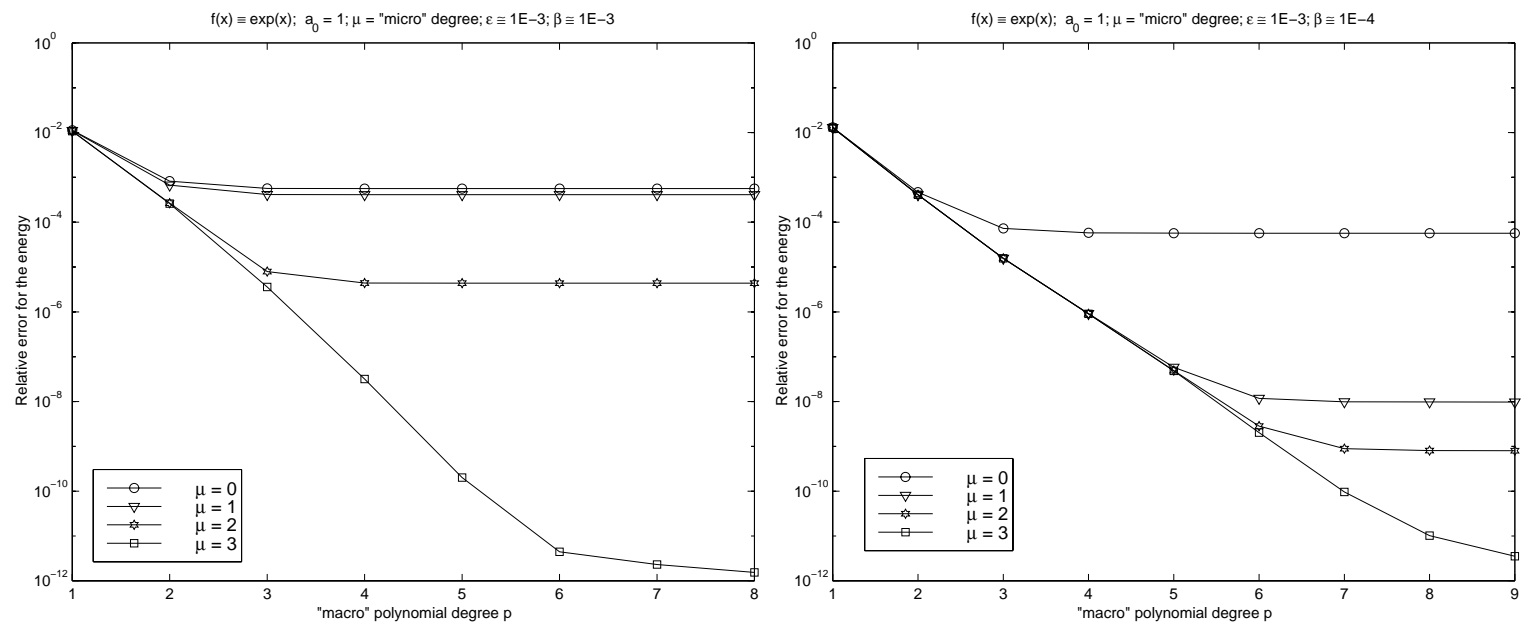

Figure 9: Exponential rate of convergence for singular perturbation

The exponential error decay with respect to the 'macro' $\left(p_{K}=p, \forall K \in \mathcal{T}\right)$, respectively 'micro' ( $\mu_{K}=\mu$ for $K \in \mathcal{T}_{\text {int }}$ ) degree is shown in Fig. 9 for $\varepsilon \cong 10^{-3}$ and $\beta \cong 10^{-3}, \beta \cong 10^{-4}$, respectively. As before, in elements near the boundary $\left(K \in \mathcal{T}_{b}\right)$ we omit the microscale shape functions (i.e. $\mu_{K}=0$ ), while in the interior element $K \in \mathcal{T}_{\text {int }}$ we choose $\mu_{K}=0, \ldots, 3$.

Our experiments show clearly that the generalized $p$-FEM performs equally well for $\beta=1$ and $\beta=10^{-3}, 10^{-4}$, over a wide range of $\varepsilon$, from $\beta / \varepsilon>>1$ to $\beta / \varepsilon<<1$. We emphasize that asymptotic expansions for the limiting cases $\beta / \varepsilon \rightarrow 0$ or $\varepsilon / \beta \rightarrow 0$ differ substantially, as do the limits. The generalized FEM performs robustly over the whole range of $\beta / \varepsilon$.

\section{References}

[1] I. Babuška, B. Q. Guo, Regularity of the solution of elliptic problems with piecewise analytic data. Part 1: Boundary value problems for linear elliptic equation of second order, SIAM J. Math. Anal., 19 (1988), pp. 172-203.

[2] I. Babuška, G. Caloz, J. Osborn, Special finite element methods for a class of second order elliptic problems with rough coefficients, SIAM J. Numer. Anal., 31 No. 4, (1994), pp. 945-981.

[3] R. C. Morgan, I. Babuška, An approach for constructing families of homogenized equations for periodic media. I: An integral representation and its consequences, SIAM J. Math. Anal. Vol. 22, No. 1 (1991) pp. 1-15. II: Properties of the kernel, SIAM J. Math. Anal. Vol. 22, No. 1 (1991) pp. 16-33.

[4] T. Hou, X.-H. Wu, Z. Cai, Convergence of a multiscale finite element method for elliptic problems with rapidly oscillating coefficients, (to appear in Math. Comp.).

[5] O. A. Oleinik, A. S. Shamaev, G. A. Yosifian, Mathematical Problems in Elasticity and Homogenization, North-Holland (1992). 
[6] A. Bensoussan, J.L. Lions and G. Papanicolau, Asymptotic Analysis for Periodic Structures, North Holland, Amsterdam (1978).

[7] A.M. Matache, Spectral and p-Finite Elements for homogenization problems, Doctoral Dissertation (in preparation).

[8] A.M. Matache, I. Babuška and C. Schwab, Generalized p-FEM in homogenization, Report 99-01, Seminar for Applied Mathematics, ETH Zürich, Switzerland.

[9] J.M. Melenk, On n-widths for elliptic problems, Report 98-02, Seminar for Applied Mathematics, ETH Zürich, Switzerland (in press in J. Math. Anal. Appl.). 